\title{
Parallel two-wheel vehicle with underslung vehicle body
}

\author{
Yukinori SAGO*, Yoshiyuki NODA**, Kiyoaki KAKIHARA*** and Kazuhiko TERASHIMA* \\ * Department of Mechanical Engineering, Toyohashi University of Technology \\ 1-1 Hibarigaoka, Tempaku-cho, Toyohashi-shi, Aichi 441-8580, Japan \\ E-mail: sagou@syscon.me.tut.acjp \\ ** Department of Mechanical Engineering, University of Yamanashi \\ 4-3-11 Takeda, Kofu-shi, Yamanashi 400-8511, Japan \\ *** KER Co., Ltd. \\ 215 Toyogaoka-cyo, Toyokawa-shi, Aichi 442-0808, Japan
}

Received 10 March 2014

\begin{abstract}
This paper presents an advanced parallel two-wheel vehicle that has an underslung vehicle body. In the proposed parallel two-wheel vehicle, since large wheels which its diameter is $1.05[\mathrm{~m}]$ are applied, and the battery, the actuators and the controller are placed at the lower position in the vehicle body, the gravity center of vehicle body with a passenger can be assigned at the lower position than the wheel axis. Therefore, the vehicle has a pendular structure that enables the vehicle body with the passenger to always maintain the stable posture, even if the vehicle is in the power-off or control-off condition. A 2-DOF joystick that has operation in pitching and yawing is applied to the proposed vehicle. The elderly or handicapped passenger can operate easily and intuitively the vehicle by this joystick. Moreover, in order to suppress the sway of the vehicle on the pitch axis of the vehicle body while vehicle is driven, the sway suppression control system with an active mass system is proposed in this paper. The control system of the active mass system is designed by a backstepping method. The effectiveness of the proposed pitch sway suppression control system with the active mass system is verified by the experiments using the proposed parallel two-wheel vehicle with an underslung vehicle body.
\end{abstract}

Key words : Parallel two-wheel vehicle, Active mass system, Lower gravity center, 2-DOF joystick, Seat positioning, Pitch sway suppression, Backstepping method

\section{Introduction}

In recent years, barrier-free society has gained traction, and the welfare environment has been gradually improved. There have been many studies on an electric wheelchair such as welfare device for the elderly and people with lower physical ability. Demand for the electric wheelchair is expected to increase in the future. In addition, special attention has been paid to personal vehicle with low energy requirement, which is required to support for short-distance transport(Lim,et al., 2008),(Ueno,et al., 2009),(Noda,et al., 2010).

The typical electric wheelchair is driven with four-wheel composed of the front casters and the rear wheels. However, since these wheelchairs have large turning radius, it is difficult to pass through in a narrow passage. A parallel two-wheel vehicle with structure of inverted pendulum, which has small turning radius, has been developed in recent years(Sasaki,et al., 2005),(Nakagawa,et al., 2011),(Shibata and Ichii, 2013),(Karkoub and Parent, 2004),(Ahmad,et al., 2011),(Li and Ando, 2013). In such vehicles, an inverted pendulum control system maintains the vehicle's stability by only using the two driving parallel wheels. Therefore, the gravity center of the vehicle body with a passenger is higher than the wheel axis, and the vehicle is moved by leaning the vehicle body forward or backward by moving the gravity center. However, in the event that the vehicle is in the power-off or control-off status caused by a breakdown in the vehicle, the stability of the vehicle cannot be maintained. Moreover, we are now considering such the vehicle that the passengers focused on not only young people but else elderly and handicapped people can drive with sitting attitude. Then, in this case, since the gravity center is lower than the standing posture, the larger action of the passenger's upper body is required for operating the vehicle. However, the elderly or handicapped passenger is difficult to behave the large action in the vehicle. 


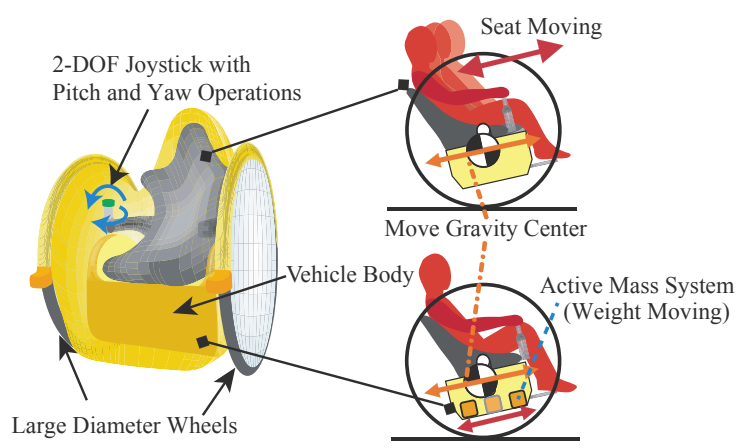

(a) Illustration of Proposed Novel Vehicle

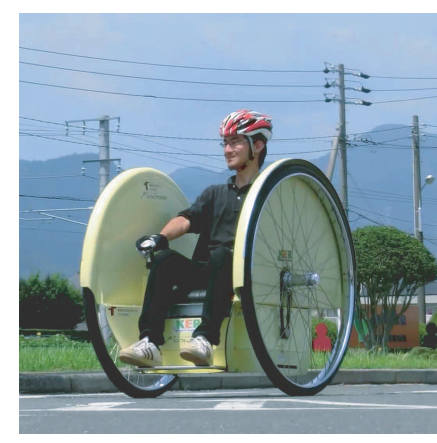

(b) Vehicle Overview

Fig. 1 Proposed Parallel Two-wheel Vehicle with Unslung Vehicle Body

Therefore, we propose a novel parallel two-wheel vehicle with safety and easy operation which can be used by elderly and handicapped people. The proposed vehicle has the lower gravity center of the vehicle body as shown in Fig.1. Because the vehicle has the large diameter wheels and the actuators, the battery and the control devices are placed under the wheel axis, the vehicle's gravity center with the passenger seated is designed to be lower than the wheel axis. Therefore, stability of the vehicle is maintained stable without electric power supply. 2-DOF joystick that has pitch and yaw rotation mechanism is installed in the proposed vehicle that has back-and-forth motion and yaw rotation. Using the novel joystick, the passenger can operate the vehicle intuitively.

However, the vehicle body is leaned by shifting the center of gravity according to the change of his seated posture when the passenger is sitting to the proposed vehicle. In the proposed vehicle, the posture of the vehicle body with the passenger is compensated by automatically adjusting the position of the passenger is seat. Moreover, in order to suppress the sway of the vehicle body on the pitch axis while driving the vehicle, the sway suppression control system of the vehicle body is newly proposed in this study. In the control system, an active mass system is installed inside the vehicle body(Noda,et al., 2012). In the design of the control system, the vehicle body dynamics is modelled using the Lagrange equation of motion, and, the control system of the pitch sway suppression control with the active mass system is designed by a backstepping method(Krsti and Tsiotras, 1999),(Fu and Zao, 2007).

The positioning control of the weight center of the vehicle has been proposed in previous studies(Kato,et al., 2011). Before the vehicle is traveling, the static attitude angle of the vehicle body is controlled by the seat positioning control system that was constructed by mechanism of shifting passenger's gravity center. Therefore, the pitch sway of the vehicle body while driving the vehicle cannot be suppressed by the previous control system. This paper presents that the pitch sway can be suppressed by using the proposed active mass system. The effectiveness of the proposed pitch sway suppression control system is verified by experiments using the proposed parallel two-wheel vehicle with the underslung vehicle body.

\section{Overview of Parallel Two-wheel Vehicle with Underslung Vehicle Body}

The parallel two-wheel vehicle with lower gravity center consists of two large diameter driving wheels and the vehicle body where the passenger sits on as shown in Fig.1(a). The power-supply, control devices and actuators are assigned on the lower position than the wheel axis. As a result, the gravity center of this vehicle with the passenger sitting on the seat is lower than the wheel axis. Therefore, it allows the vehicle to always keep stably without electric power supply. A conventional parallel two-wheel vehicle's gravity center with the passenger is higher position than the wheel axis, and an inverted pendulum control system is applied for stably keeping the vehicle body and operating the vehicle by moving the gravity center of the passenger. However, in the proposed vehicle, the moment of force by the passenger moving is small, because the gravity center of the sitting passenger is assigned to near the wheel axis. Accordingly, it is difficult to operate the vehicle by shifting the gravity center of the passenger. Therefore, the two-wheel vehicle is operated by the 2-DOF joystick. This joystick has both pitch and rotation operations, and the vehicle is corresponding to drive the vehicle in back and forth direction and yaw rotation. It is a human-friendly interface with easiness to operate the vehicle, since the movement of the joystick is the same as the movement of the vehicle.

When the passenger sits on the seat of the vehicle, the vehicle body is leaned statically by moving the gravity center of the vehicle body with the passenger. In this paper, in order to compensate the vehicle body's leaning, a seat positioning control system is mounted on the seat supporting structure of the vehicle body. And, while the vehicle is driven, the 
Table 1 Specification of Vehicle

\begin{tabular}{|c|c|c|c|c|c|}
\hline Mass [kg] & Size [m] & Wheel Radius [m] & Wheel Distance [m] & Driving Voltage [V] & Driving Power [W] \\
\hline 136.7 & W0.90×D1.10×H1.08 & 0.525 & 0.802 & 24 & 300 \\
\hline
\end{tabular}

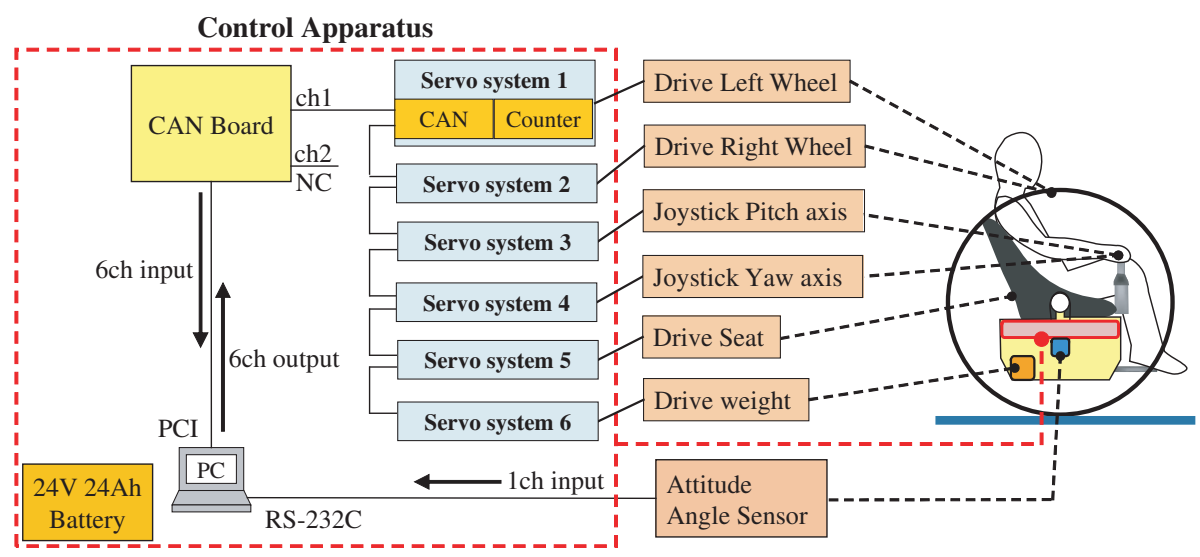

Fig. 2 Control System of Vehicle

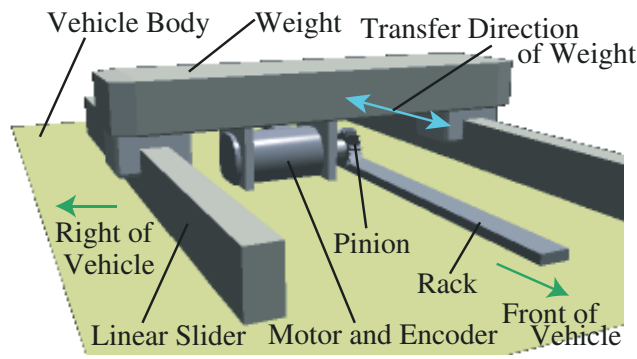

(a)Illustration of Active Mass System

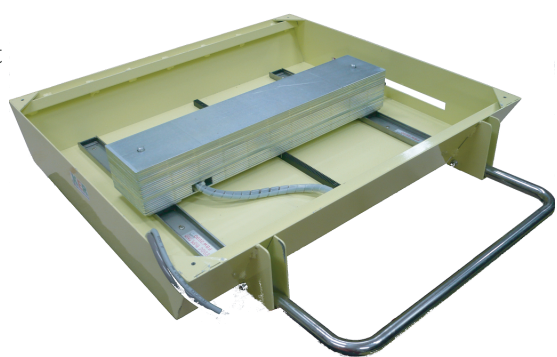

(b)Photo of Active Mass System

Fig. 3 Active Mass System

vehicle body is swayed on the pitch axis by the acceleration of the vehicle. In order to suppress the vehicle body swaying, the pitch sway suppression control system with the active mass is proposed in this study. The pitch sway suppression control system is mounted on the bottom of the vehicle body.

The parallel two-wheel vehicle with the underslung vehicle body developed by the authors is shown in Fig.1(b), and the specification of the developed two-wheel vehicle is shown in Table 1. The construction of the control system in the parallel two-wheel vehicle is shown in Fig.2. The right and left wheels are rotated by DC servomotors, reducers and pulley mechanisms. The rotations of the wheels are detected by the rotary encoders fitted on the servomotors. The pitching and yawing of the joystick are detected by the rotary encoders fitted on each axis. The joystick is able to indicate the reaction force haptically by the DC servomotors installed in the joystick. The weight in the active mass system is transferred by DC servomotor, reducer, rack-and-pinion and linear sliders as shown in Fig.3. The position of the weight is detected by the rotary encoder fitted on the servomotor. The seat transfer system is also the same mechanism as the active mass system. The pitch angle and the angular velocity of the vehicle body are detected by the attitude angle sensor located on the bottom of the vehicle body.

The signals detected by the rotary encoders are collected in the PC through CAN board and the counter boards of the servo system. And, the signals detected by the attitude angle sensor are collected in the PC through the RS-232c interface. Then, the input signals are added to the servomotors through the CAN board.

\section{Vehicle Operation by 2-DOF Joystick with Pitch and Yaw Axes}

For intuitive operation of the parallel two-wheel vehicle, 2-DOF joystick with both pitch and yaw operations as shown in Fig.4 is developed in this study. The operation of the joystick is the same as the vehicle motion that consists of back-and-forth motion and yaw rotation. The relation between the joystick operation and the vehicle motion is shown as

$$
\left[\begin{array}{c}
F_{v} \\
T_{v}
\end{array}\right]=\left[\begin{array}{cc}
F_{v \max } / \psi_{y j o y \max } & 0 \\
0 & T_{v \max } / \psi_{z j o y \max }
\end{array}\right]\left[\begin{array}{l}
\psi_{y j o y} \\
\psi_{z j o y}
\end{array}\right]
$$




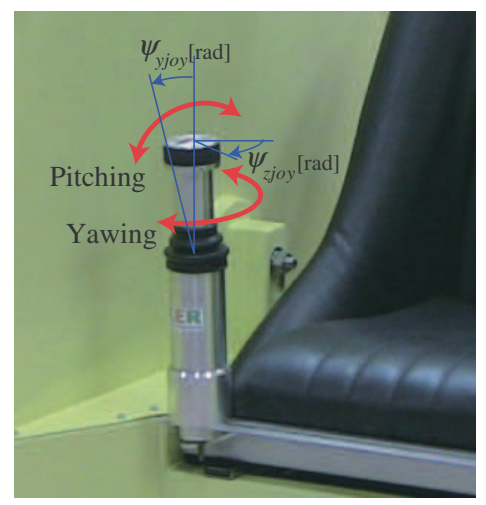

(a) Operation of Joystick

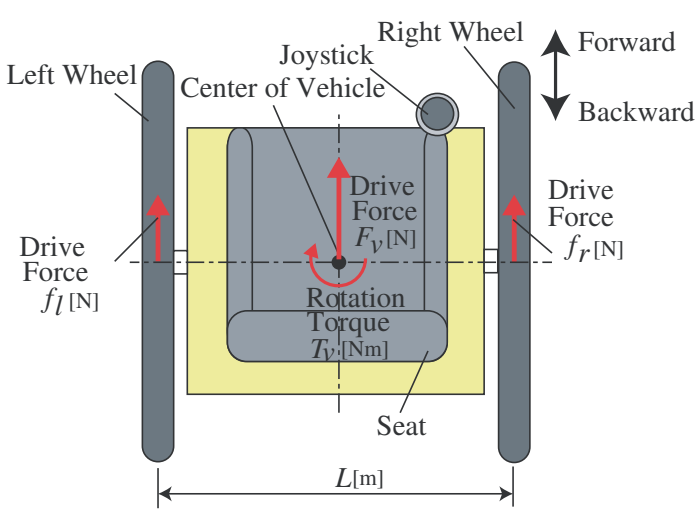

(b) Motion of Vehicle (Top View)

Fig. 4 Vehicle Operation by Joystick with Pitch and Yaw Operations

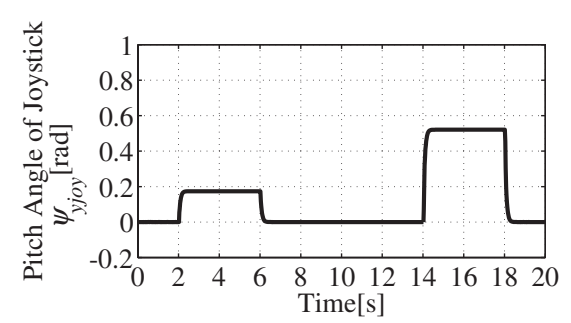

(a) Time Series Graph of Pitch Angle of Joystick

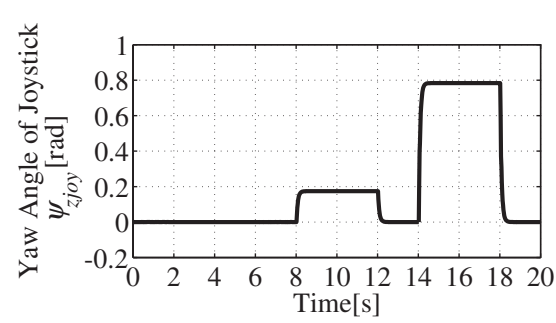

(b) Time Series Graph of Yaw Angle of Joystick

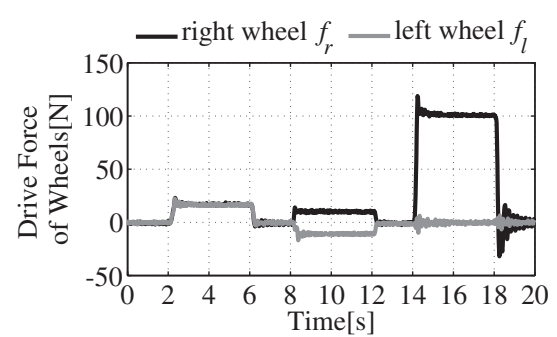

(c) Time Series Graph of Drive Force of Wheels

Fig. 5 Experimental Results of Drive Forces to Joystick Operation

where $F_{v}[\mathrm{~N}]$ and $T_{v}[\mathrm{Nm}]$ are the propulsion force and the rotation torque of the vehicle, respectively. $\psi_{y j o y}[\mathrm{rad}]$ and $\psi_{z j o y}[\mathrm{rad}]$ are the pitch angle and the yaw angle of the joystick, respectively. The subscript 'max' represents the maximum of each variable, and is obtained as $F_{v \max }=100.7[\mathrm{~N}], T_{v \max }=40.4[\mathrm{Nm}], \psi_{y j o y \text { max }}=30 \times \pi / 180[\mathrm{rad}]$ and $\psi_{z j o y \text { max }}=$ $45 \times \pi / 180[\mathrm{rad}]$. In Fig.4(b), the drive forces $f_{r}[\mathrm{~N}]$ and $f_{l}[\mathrm{~N}]$ added to the right and left wheels are shown as

$$
\left[\begin{array}{c}
f_{r} \\
f_{l}
\end{array}\right]=\left[\begin{array}{cc}
1 / 2 & 1 / L \\
1 / 2 & -1 / L
\end{array}\right]\left[\begin{array}{c}
F_{v} \\
T_{v}
\end{array}\right]
$$

where $L[\mathrm{~m}]$ is the wheel distance. $L$ is obtained as $L=0.802[\mathrm{~m}]$. Current control is used in the servomotors driving the wheels. Therefore, the relation between the drive forces $f_{r}[\mathrm{~N}]$ and $f_{l}[\mathrm{~N}]$ and the current commands $i_{r}[\mathrm{~A}]$ and $i_{l}[\mathrm{~A}]$ added to the motors is obtained by experiments and linearly-approximated. The relation is represented as

$$
\left[\begin{array}{c}
i_{r} \\
i_{l}
\end{array}\right]=\left[\begin{array}{cc}
\alpha_{r} r_{w} & 0 \\
0 & \alpha_{l} r_{w}
\end{array}\right]\left[\begin{array}{c}
f_{r} \\
f_{l}
\end{array}\right]
$$

where $\alpha_{r}[\mathrm{~A} / \mathrm{Nm}]$ and $\alpha_{l}[\mathrm{~A} / \mathrm{Nm}]$ are the proportional gains to drive the right and the left wheels, respectively. In this paper, the proportional gains are obtained as $\alpha_{r}=0.0946$ and $\alpha_{l}=0.0911 . r_{w}[\mathrm{~m}]$ is the radius of the wheel, and is obtained as $r_{w}$ $=0.525[\mathrm{~m}]$. By summarizing from Eqs.(1)-(3), the current commands generated by the joystick operation are represented as

$$
\left[\begin{array}{c}
i_{r} \\
i_{l}
\end{array}\right]=\left[\begin{array}{cc}
F_{v \max } \alpha_{r} r_{w} / 2 \psi_{y j o y \max } & T_{v \max } \alpha_{r} r_{w} / \psi_{z j o y \max } L \\
F_{v \max } \alpha_{l} r_{w} / 2 \psi_{y j o y \max } & -T_{v \max } \alpha_{l} r_{w} / \psi_{z j o y \max } L
\end{array}\right]\left[\begin{array}{c}
\psi_{y j o y} \\
\psi_{z j o y}
\end{array}\right] .
$$

In order to verify the vehicle operation, the drive forces to the right and left wheels are measured in experiments. The drive forces of both wheels are measured by digital force gauge mounted on the wheels' outer rims. The experimental results of the drive forces $f_{r}$ and $f_{l}$ to the joystick operation are shown in Fig.5. In the figure, (a) and (b) show the pitch angle and the yaw angle of the joystick, respectively. (c) shows the drive forces added to right and left wheels. In Fig.5(c), the black and the gray lines represent the drive forces of the right and the left wheels, respectively. At first in the experiment, when the joystick is operated by the pitch angle $\psi_{z j o y}=10 \times \pi / 180[\mathrm{rad}]$, the right and the left wheels are driven with the same force $16.8[\mathrm{~N}]$. Then, when the joystick is operated by the yaw angle $\psi_{y j o y}=10 \times \pi / 180[\mathrm{rad}]$, the drive force of the right wheel $11.2[\mathrm{~N}]$ and drive force of the left wheel $-11.2[\mathrm{~N}]$ arise. Finally, when the joystick is operated by the maximum pitch and yaw angles, the drive force of the right wheel $100.7[\mathrm{~N}]$ and the drive force of the left wheel $0.0[\mathrm{~N}]$ arise. It was verified that the drive force of the vehicle can be controlled at will by operating the 2-DOF joystick. 


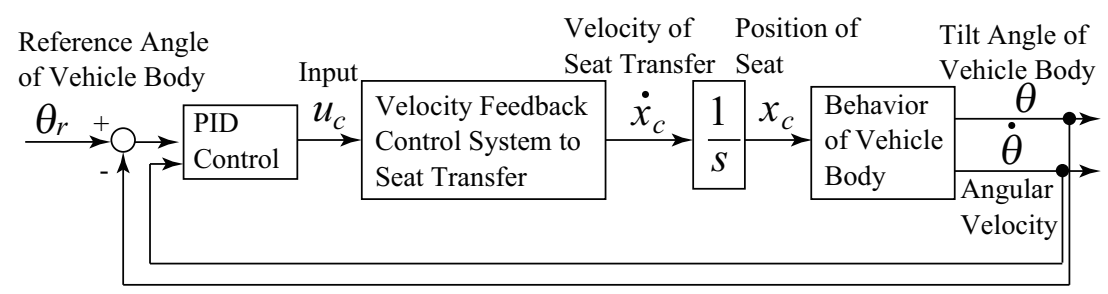

Fig. 6 Block Diagram of The Vehicle Body's Static Attitude Angle Control by Seat Positioning Control System

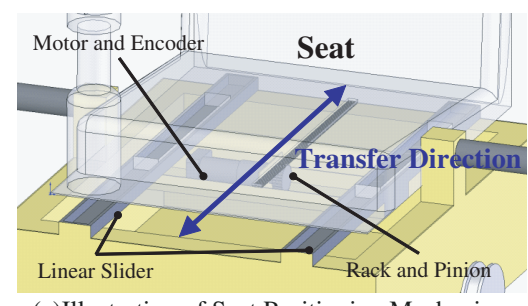

(a)Illustration of Seat Positioning Mechanism

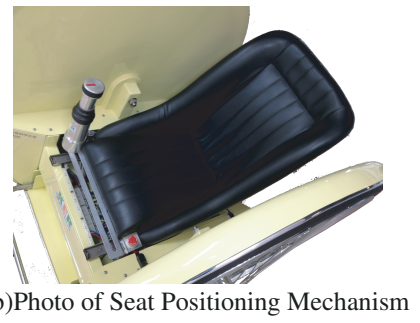

(b)Photo of Seat Positioning Mechanism

Fig. 7 Seat Positioning Mechanism

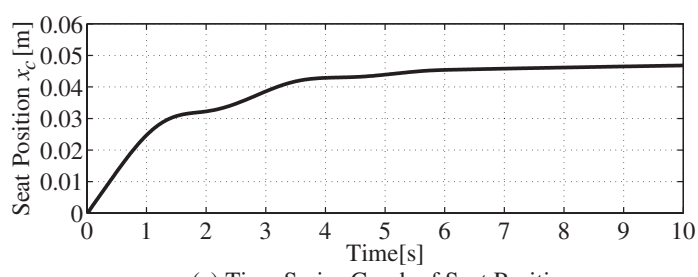

(a) Time Series Graph of Seat Position

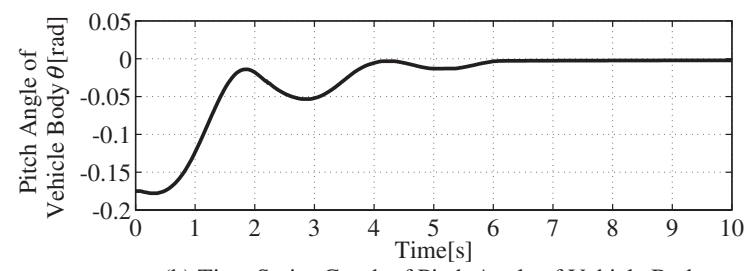

(b) Time Series Graph of Pitch Angle of Vehicle Body

Fig. 8 Experimental Results of Seat Positioning Control

\section{Seat Positioning Control System}

When the passenger sits on the seat of the vehicle, the vehicle body is leaned by moving the gravity center of the vehicle body with the passenger. For compensating the vehicle body leaning, the seat positioning control system is proposed in this paper. The vehicle body's static posture lean control can be compensated by the proposed control system. Further, the proposed control system is able to change the angle of the vehicle body in a state of stopping the vehicle. The proposed seat positioning control system is shown in Fig.6. In the control system, the pitch angle and the angular velocity of the vehicle body are detected by the attitude angle sensor. The control input to the servomotor system is generated by PID control, consisting of $\mathrm{P}$ control to compensate the error between the reference angle and the pitch angle of the vehicle body, I control to the integrated error, and D control to the angular velocity of the vehicle body. The servomotor system consists of the velocity feedback control system. The seat is transferred by driving the servomotor as shown in Fig.7. By moving the seat with the passenger, the posture of the vehicle body is compensated. In PID control, the control parameters are adjusted by performing experiments for control of static attitude angle in a short time. As the result, the proportional, the integral and the derivative gains are given as $0.233 \times 10^{-2}, 0.151$ and $0.116 \times 10^{-13}$ respectively. And, the experimental results are shown in Fig.8. In the experiment, the reference pitch angle of vehicle body is given $0[\mathrm{rad}]$ as flat, and the seat before the positioning motion is located at center. The passenger with $59.25[\mathrm{~kg}]$ weight rides on the vehicle. In Fig.8, (a) is the seat position and (b) is the pitch angle of the vehicle body. Fig.8(b) shows that the sway of the vehicle body is caused by the seat positioning motion. However, the sway is damped, because the vehicle body is structurally stable. Therefore, the vehicle body with the passenger becomes flat after 7 seconds by adjusting the seat position.

This control system works only when the vehicle is static, because of the compensation to the vehicle body's static posture. Therefore, while the vehicle is driven, the seat is secured on the vehicle body.

\section{Pitch Sway Suppression Control System of Vehicle Body}

While the vehicle is driven, the vehicle body is swayed due to the pitching oscillation by the acceleration of the vehicle driving. In order to suppress the vehicle body's swaying, the pitch sway suppression control system with an active 


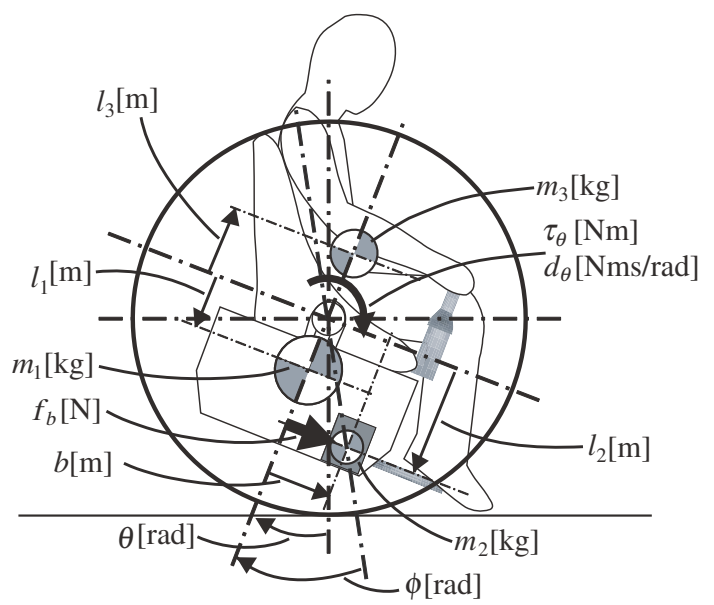

Fig. 9 Model Parameters Concerned with Vehicle Body Behavior

mass is proposed in this paper. The proposed control system that does not use the driving force of the wheel is possible to stabilize the sway of the vehicle body even if the wheel is fixed by the braking device. Therefore, proposed attitude control system is advantageous for the safety of the parallel two-wheel vehicle. For designing the control system, the dynamics of the pitch angle in relation to the vehicle's body swaying is modelled by using the Lagrange equation of motion. Then, the control system is designed by the backstepping method(Krsti and Tsiotras, 1999),(Fu and Zao, 2007).

\subsection{Modelling Behavior of Vehicle Body}

The vehicle body behavior is illustrated in Fig.9. In the figure, $m_{1}, m_{2}$ and $m_{3}[\mathrm{~kg}]$ are the mass of the vehicle body without the passenger and the weight in the active mass system, the mass of the weight in the active mass system and the mass of the passenger, respectively. And, $l_{1}, l_{2}$ and $l_{3}[\mathrm{~m}]$ are the gravity center height of the vehicle body without the passenger and the weight, the gravity center height of the weight in the active mass system and the gravity center height of the passenger, respectively. $\theta[\mathrm{rad}]$ is the pitch angle between the line connecting the gravity center of the vehicle body with the center of the wheel axis and the vertical axis. $\phi[\mathrm{rad}]$ is the angle between the line connecting the gravity center of the weight with the center of the wheel axis and that connecting the gravity center of the vehicle body with the center of the wheel axis. $b[\mathrm{~m}]$ is the horizontal position of the weight to make the vehicle body dynamically control attitude. $\tau_{\theta}[\mathrm{Nm}]$ and $d_{\theta}[\mathrm{Nms} / \mathrm{rad}]$ are the external torques around the wheel axis caused by driving the wheels and the viscous damping coefficient to rotate the wheels, respectively. The Lagrange equation of motion of the behavior of the vehicle body is shown as

$$
\frac{d}{d t}\left(\frac{\partial T}{\partial \dot{x}}\right)-\frac{\partial T}{\partial x}+\frac{\partial U}{\partial x}+\frac{\partial D}{\partial \dot{x}}=u
$$

where $T$ is the kinetic energy, $U$ is the potential energy, and $D$ is the dissipative energy. The generalized coordinate is represented as $x=\left[\begin{array}{ll}\theta & b\end{array}\right]$. These energies are represented as

$$
\begin{aligned}
& T=\frac{1}{2}\left(m_{1} l_{1}^{2}+m_{2}\left(l_{2}^{2}+b^{2}\right)+m_{3} l_{3}^{2}\right) \dot{\theta}^{2}+\frac{1}{2} m_{2} \dot{b}^{2}, \\
& D=\frac{1}{2} d_{\theta} \dot{\theta}^{2}, \\
& U=m_{1} g l_{1}(1-\cos \theta)+m_{2} g \sqrt{l_{2}^{2}+b^{2}}(1-\cos (\theta-\phi))+m_{3} g l_{3}(1+\cos \theta),
\end{aligned}
$$

where $g\left[\mathrm{~m} / \mathrm{s}^{2}\right]$ is the gravity acceleration. By substituting Eqs.(6)-(8) into Eq.(5), the differential equation can be obtained as

$$
\begin{aligned}
& A_{1} \ddot{\theta}+A_{2} \dot{\theta}+A_{3} \sin \theta-m_{2} g b \cos \theta=\tau_{\theta}, \\
& B_{1} \ddot{b}+B_{2} b-m_{2} g \sin \theta=f_{b},
\end{aligned}
$$

where

$$
A_{1}=m_{1} l_{1}^{2}+m_{2}\left(l_{2}^{2}+b^{2}\right)+m_{3} l_{3}^{2}, A_{2}=2 m_{2} b \dot{b}+d_{\theta}, A_{3}=\left(m_{1} l_{1}+m_{2} l_{2}-m_{3} l_{3}\right) g, B_{1}=m_{2}, B_{2}=-m_{2} \dot{\theta}^{2}+\frac{m_{2} g}{\sqrt{l^{2}+b^{2}}} .
$$




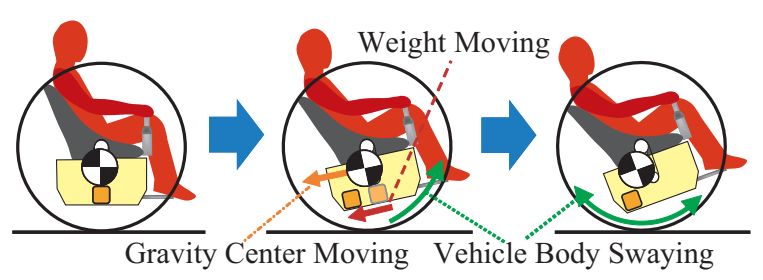

Fig. 10 Experiment Procedure for Model Parameters Identification

Table 2 Model parameters

\begin{tabular}{|c|c|c|c|c|c|c|c|}
\hline Notation & $l_{1}[\mathrm{~m}]$ & $l_{2}[\mathrm{~m}]$ & $l_{3}[\mathrm{~m}]$ & $m_{1}[\mathrm{~kg}]$ & $m_{2}[\mathrm{~kg}]$ & $m_{3}[\mathrm{~kg}]$ & $d_{\theta}[\mathrm{Nms} / \mathrm{rad}]$ \\
\hline Values & 0.301 & 0.370 & 0.317 & 91.30 & 26.70 & 59.25 & 25.313 \\
\hline
\end{tabular}

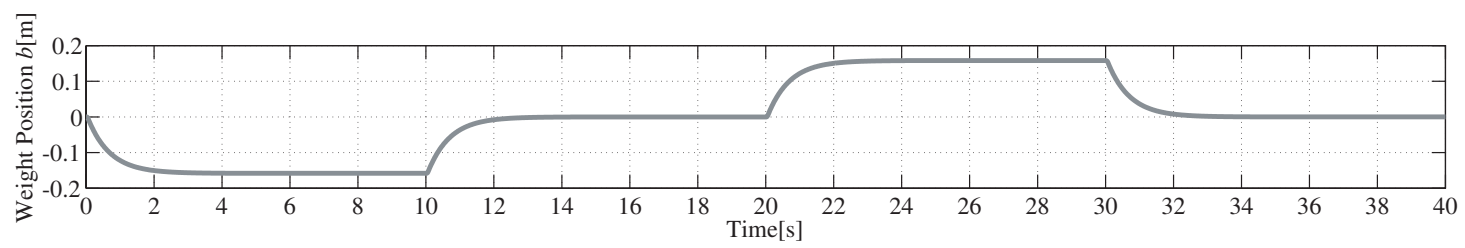

(a) Time Series Graph of Weight Position

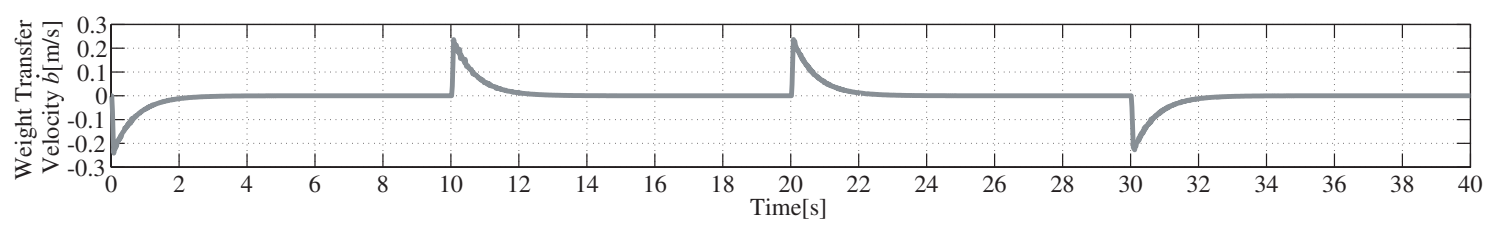

(b) Time Series Graph of Weight Transfer Velocity

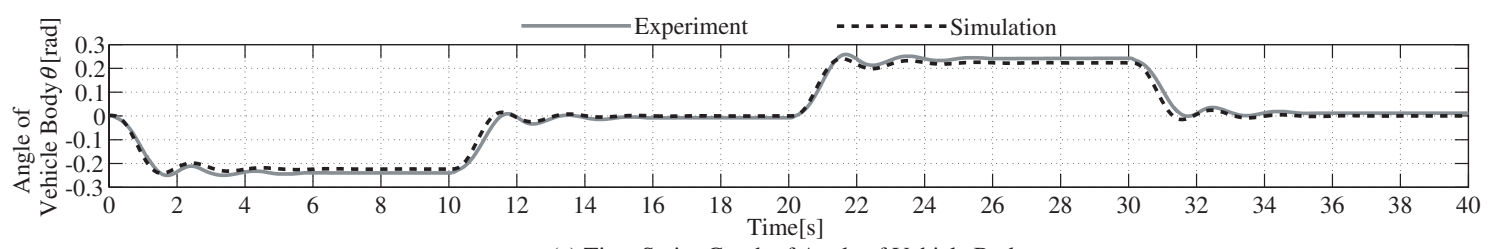

(c) Time Series Graph of Angle of Vehicle Body

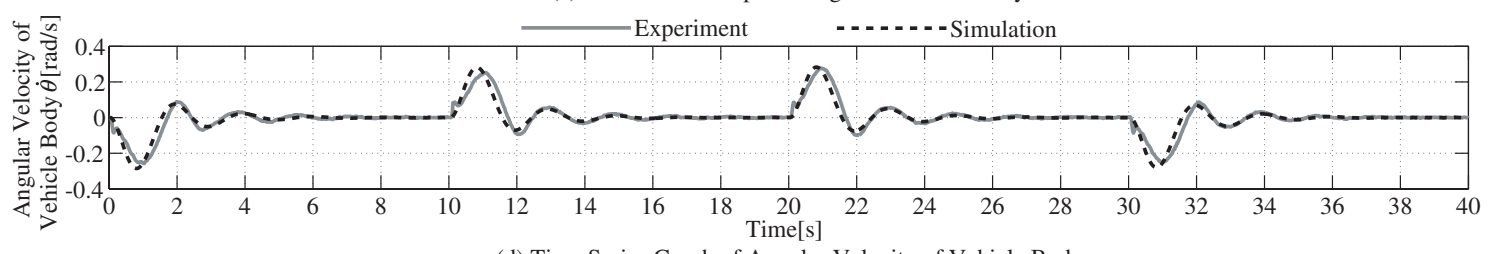

(d) Time Series Graph of Angular Velocity of Vehicle Body

Fig. 11 Comparison between Behaviors of Vehicle Body in Experiment and Simulation

\subsection{Identification of Model Parameters}

In the model of the behavior of the vehicle body presented in the previous section, $\tau_{\theta}$ and $f_{b}$ are the external torques, namely, the torque generated by the servomotors for driving the wheels and the driving force to the weight in the active mass system, respectively. However, we assume that the reaction force that is applied to the vehicle body by $f_{b}$ is not included in $\tau_{\theta}$ because the ratio of $m_{2}$ to the total weight is low. The pitch angle $\theta$, the angular velocity $\dot{\theta}$ of the vehicle body, the position $b$ and the velocity $\dot{b}$ of the weight are the state variables in the model. The mass $m_{1}, m_{2}$ and $m_{3}$, the height $l_{1}, l_{2}$ and $l_{3}$, and the viscous damping coefficient $d_{\theta}$ are the constant parameters. The mass $m_{1}, m_{2}$ and $m_{3}$ are measured by a weigher. The parameters $l_{1}, l_{2}, l_{3}$ and $d_{\theta}$ are the unknown parameters, and are identified by minimizing the error between the vehicle body's behavior in the experiment and the simulation. In the experiment for the identification, the weight in the active mass system is moved as shown in Fig.10. Then, the vehicle body is swayed by moving the weight. The weight position feedback control system has been constructed to the active mass system. The parameters are searched by minimizing the error using a downhill simplex method(Nelder and Mead, 1965).

The identified model parameters are shown in Table 2. The behaviors of the vehicle body in the experiment and in the simulation are compared in Fig.11. Here, (a) and (b) show the position and the velocity of the weight transfer in the active mass system, and (c) and (d) show the tilt angle and the angular velocity of the vehicle body. In Fig.11(c) and (d), the solid lines are the experimental results, and the broken lines are the simulation results. As seen from Fig.11, the 
sway of the vehicle body can be represented precisely by the proposed model. Therefore, the proposed model is used for designing the pitch sway suppression control system with the active mass system.

\subsection{Design of Pitch Sway Suppression Control Based on Backstepping Method}

In order to simply design the sway suppression control system, the model of behavior of the vehicle body is simplified by linearization. It is assumed that the pitch angle to sway the vehicle body is small. Eqs.(9) and (10) are linearized as

$$
\begin{aligned}
& A_{1} \ddot{\theta}+A_{2} \dot{\theta}+A_{3} \theta-m_{2} g b=\tau_{\theta} . \\
& B_{1} \ddot{b}+B_{2} b+m_{2} g \theta=f_{b},
\end{aligned}
$$

The position feedback control system using PID control is applied for positioning the weight in the active mass system. Therefore, it is assumed that the disturbance $B_{2} b+m_{2} g \theta$ in Eq. 12 is damped and the weight position $b$ tracks quickly to the reference position $b_{r e f}[\mathrm{~m}]$ such as $b \approx b_{\text {ref }}$. Moreover, Eq.(11) is transformed by polynomial deformation to the spring-mass-damper system as

$$
M(b) \ddot{\theta}+C(b, \dot{b}) \dot{\theta}+K \theta=d\left(\tau_{\theta}\right)+u(b),
$$

where

$$
\begin{aligned}
& M(b)=m_{1} l_{1}^{2}+m_{2}\left(l_{2}^{2}+b^{2}\right)+m_{3} l_{3}^{2}, \\
& C(b, \dot{b})=2 m_{2} b \dot{b}+d_{\theta}, \\
& K=\left(m_{1} l_{1}+m_{2} l_{2}-m_{3} l_{3}\right) g, \\
& d\left(\tau_{\theta}\right)=\tau_{\theta}, u(b)=m_{2} g b .
\end{aligned}
$$

In Eq.(13), $\tau_{\theta}$ is the external torque to sway the vehicle body, and is treated as the disturbance $d$ in the control system design. $b$ is the weight position, and the torque $m_{2} g b$ generated by the weight position is treated as the control input $u$ to stabilize the posture of the vehicle body.

Here, the model in Eq.(13) is a Linear Parameter Varying (LPV) system, and has two control variables $\theta$ and $\dot{\theta}$. Many control approaches for the LPV system have been proposed in the previous studies(Rugh and Shamma, 2000). In this paper, the backstepping method, which is one of the control approaches for the nonlinear system, is applied to design the pitch sway suppression control. It enables us to design the LPV system or nonlinear system with small number of the control variables easily. The backstepping method is the useful control design such that a Lyapunov function is sequentially-constructed with respect to each state variable based on the formal Lyapunov function as

$$
U_{(x, y)}=V_{(x)}+\frac{1}{2}\left[y-\mu_{(x)}\right]^{2}
$$

where $x$ is the state variable, $y$ is the controlled variable. In this paper, the state variable $x$ consists of $(\theta, \dot{\theta})$, and the controlled variable $y$ is the tilt angle $\theta$ of the vehicle body. Furthermore, the backstepping method is able to stifle the influence of disturbance and the modeling error by using nonlinear damping term. Its features are useful control design method to the proposed vehicle that road surface condition and passenger will be changed. In the first step, based on Eq.(14), the candidate Lyapunov function to the state variable $\theta$ in the model is given as

$$
V_{1}=\frac{1}{2} z_{1}^{2}=\frac{1}{2}\left(y-y_{r}\right)^{2},
$$

where $y_{r}$ is the reference pitch angle of the vehicle body, and $z_{1}$ is the error between the actual and reference pitch angles. In this paper, the reference $y_{r}$ is 0 , because the purpose of the control system is stabilization of the vehicle body in the standing posture. Thus, the Lyapunov function in Eq.(15) is represented as

$$
V_{1}=\frac{1}{2} z_{1}^{2}=\frac{1}{2} y^{2} .
$$

The time derivative of Eq.(16) is shown as

$$
\dot{V}_{1}=z_{1} \dot{z}_{1}=y \dot{y} .
$$

Here, since $\dot{z}_{1}=\dot{y}=\dot{\theta}$, Eq.(15) is replaced as

$$
\dot{V}_{1}=z_{1} \dot{z}_{1}=y \dot{y}=y \dot{\theta} .
$$


By arranging $\dot{\theta}$ as

$$
\dot{\theta}=-c_{1} y,\left(c_{1}>0\right),
$$

Eq.(18) is represented as

$$
\dot{V}_{1}=-c_{1} y^{2}<0
$$

Therefore, it can be obtained as a certain Lyapunov function. Here, the ideal state variable $\alpha$ to the state variable $\dot{\theta}$ is introduced as

$$
\alpha=-c_{1} y
$$

In the second step, the error $z_{2}$ between the actual state $\dot{\theta}$ and the ideal state $\alpha$ is shown as

$$
z_{2}=\dot{\theta}-\alpha
$$

Based on Eq.(14), the candidate Lyapunov function $V_{2}$ involving the state $z_{2}$ is given as

$$
V_{2}=V_{1}+\frac{1}{2} z_{2}^{2}
$$

The time derivative of Eq.(23) is shown as

$$
\dot{V}_{2}=\dot{V}_{1}+z_{2} \dot{z}_{2}
$$

Here, by substituting Eqs.(21) and (22) into Eq.(18), the time derivative of the Lyapunov function $\dot{V}_{1}$ is represented as

$$
\dot{V}_{1}=y\left(z_{2}+\alpha\right)=y\left(z_{2}-c_{1} y\right)=-c_{1} y^{2}+y z_{2} .
$$

And, the model shown in Eq.(13) and the time derivative of the ideal state $\alpha$ in Eq.(21) are substituted into the time derivative of Eq.(22) as

$$
\dot{z}_{2}=\ddot{\theta}-\dot{\alpha}=\ddot{\theta}+c_{1} \dot{y}=\frac{1}{M(b)} u(b)-\left(\frac{C(b, \dot{b})}{M(b)}-c_{1}\right) \dot{\theta}-\frac{K}{M(b)} \theta,
$$

where the disturbance $d$ is removed from the model of Eq.(13) for substituting. By substituting Eqs.(25) and (26) into Eq.(24), $\dot{V}_{2}$ is represented as

$$
\begin{aligned}
& \dot{V}_{2}=-c_{1} y^{2}+y z_{2}+z_{2} \dot{z}_{2}=-c_{1} y^{2}+z_{2}\left(\dot{z}_{2}+\theta\right) \\
& =-c_{1} y^{2}+z_{2}\left(\frac{1}{M(b)} u(b)-\left(\frac{C(b, \dot{b})}{M(b)}-c_{1}\right) \dot{\theta}-\left(\frac{K}{M(b)}-1\right) \theta\right) .
\end{aligned}
$$

By arranging $-c_{2} z_{2}$ as

$$
-c_{2} z_{2}=\frac{1}{M(b)} u(b)-\left(\frac{C(b, \dot{b})}{M(b)}-c_{1}\right) \dot{\theta}-\left(\frac{K}{M(b)}-1\right) \theta,\left(c_{2}>0\right),
$$

$\dot{V}_{2}$ is represented as

$$
\dot{V}_{2}=-c_{1} y^{2}-c_{2} z_{2}^{2}<0
$$

Therefore, the state variables $\theta$ and $\dot{\theta}$ are in Lyapunov stability by constructing the Lyapunov function $V_{2}$. By substituting Eqs.(21) and (22) into Eq.(28), the control input $u$ is obtained as

$$
u(b)=\left(C(b, \dot{b})-M(b)\left(c_{1}+c_{2}\right)\right) \dot{\theta}+\left(K-M(b)\left(1+c_{1} c_{2}\right)\right) \theta
$$

Since $u(b)=m_{2} g b$ as shown in Eq.(13), the weight position $b$ with the state feedback law is derived as

$$
b=\frac{1}{m_{2} g}\left\{\left(C(b, \dot{b})-M(b)\left(c_{1}+c_{2}\right)\right) \dot{\theta}+\left(K-M(b)\left(1+c_{1} c_{2}\right)\right) \theta\right\}
$$

In practical use, the weight position $b$ is realized by giving the reference weight position $b_{r e f}$ to the position feedback control system. The block diagram of the pitch sway suppression control system with the active mass system designed by the backstepping method is shown in Fig.12. The input torque $u$ is calculated using the pitch angle and the angular velocity measured by the attitude angle sensor. The reference weight position $b_{\text {ref }}$ is derived from the input torque, and the weight 


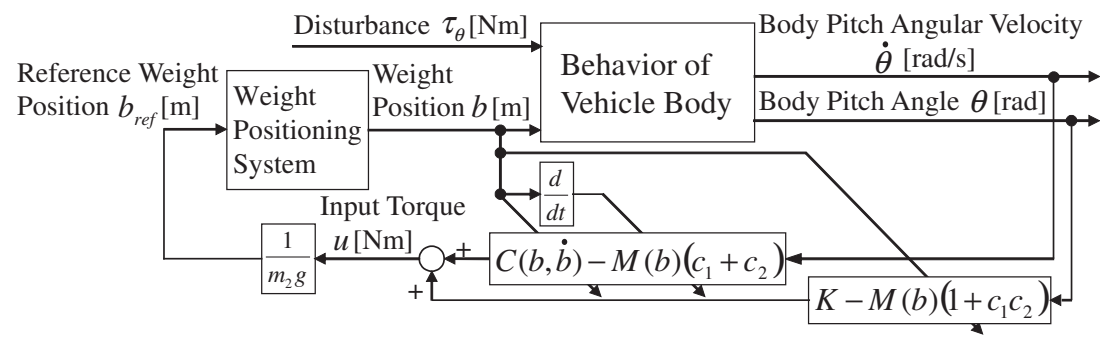

Fig. 12 Block Diagram of Pitch Sway Suppression Control System

position $b$ is realized by the weight positioning system in the active mass system. The weight position $b$ is measured by the rotary encoder fitted on the servomotor in the active mass system. $\dot{b}$ is derived as the approximate derivative of the weight position $b$ measured by the rotary encoder.

In this pitch sway suppression control system, the pitch angle $\theta$ and the angular velocity $\dot{\theta}$ of the vehicle body are converged fast with increasing the design parameters $c_{1}$ and $c_{2}$ as seen from Eq.(29). However, the control system with increased design parameters is influenced by the noise in the signal measured by the sensors. Moreover, it is hard to track the actual weight position in the active mass system to the ideal weight position generated by the control law in the sway suppression control system. In this paper, the design parameters are adjusted by performing experiments and the simulations for dynamic attitude angle convergence and suppression in a short time. As the result, the design parameters are obtained as $c_{1}=3.54$ and $c_{2}=3.54$.

\section{Experimental Results}

In order to verify the effectiveness of the proposed pitch sway suppression control system with the active mass system, experiments using the proposed parallel two-wheel vehicle were performed. In experiments, the behavior of the vehicle body with the pitch sway suppression control system is compared with that without the pitch sway suppression control system. In the vehicle without the pitch sway suppression control system, the weight in the active mass system stays at the center of the vehicle body. The experimental results are shown in Fig.13. In the figure, (a) shows the reference of the driving force added to each wheel, when (b) and (c) show the position and the velocity of the weight transfer in the active mass, respectively; (d) and (e) show the pitch angle and the angular velocity of the vehicle body, respectively. In Fig.13(b)-(e), the black and the gray lines are respectively the experimental results by the vehicle with and without the pitch sway suppression control. As seen from Fig.13(d), the sway angle of the vehicle body in the vehicle without the pitch sway suppression control is over $15.0 \times \pi / 180[\mathrm{rad}]$. On the other hand, the pitch angle in the vehicle with the pitch sway suppression control can be suppressed within $7.6 \times \pi / 180[\mathrm{rad}]$. Moreover, the residual vibration of the sway after driving the wheels is also suppressed by the proposed pitch sway suppression control. Therefore, the proposed pitch sway suppression control is useful from the viewpoint of the safety of the parallel two-wheel vehicle with the underslung vehicle body.

\section{Conclusions}

In this paper, the novel parallel two-wheel vehicle with lower gravity center has been proposed. It has the advantage of being safer than a conventional two-wheel vehicle with an inverted pendulum structure, because the proposed vehicle has a stable structure in which the gravity center of the vehicle body is lower than the wheel axis. In addition, the 2DOF joystick which has pitch and yaw operations is applied to the proposed vehicle for intuitive operation. In order to compensate the vehicle body's leaning when the passenger is seated in the vehicle, the seat positioning control system by PID control is proposed. Moreover, in order to suppress the pitch sway of the vehicle body while the vehicle is driven, a pitch sway suppression control system with an active mass system is proposed. The control system is designed by the backstepping method, and its stability is proved by finding a suitable Lyapunov function. By means of experiments using the proposed two-wheel vehicle, it has been shown that the proposed sway suppression control system is effective in the sway suppression of the vehicle body, and useful from the viewpoint of safety of the proposed two-wheel vehicle.

As ongoing problem to the improvement of the proposed vehicle's safety and stability, we must clarify the stability of the proposed vehicle when the driving force, road surface condition and passenger are changed dynamically or statically. 


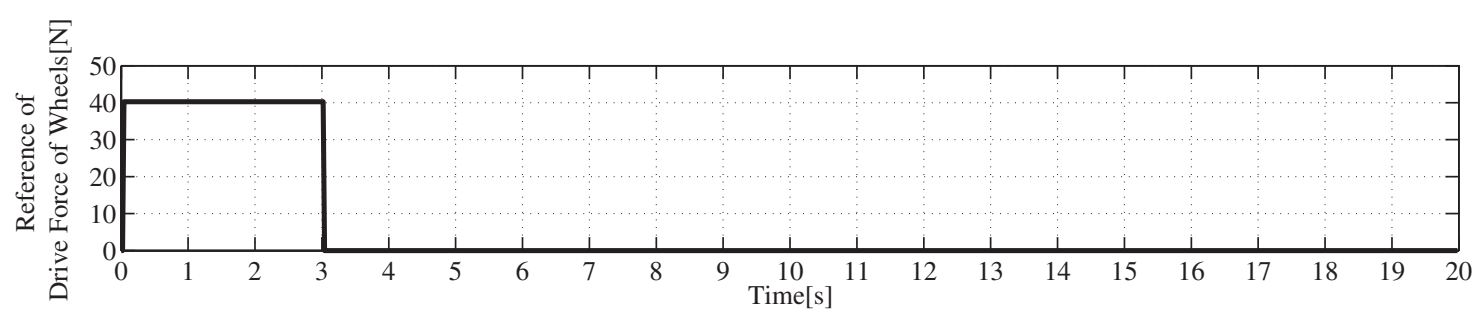

(a) Time Series Graph of Reference of Drive Force of Wheels

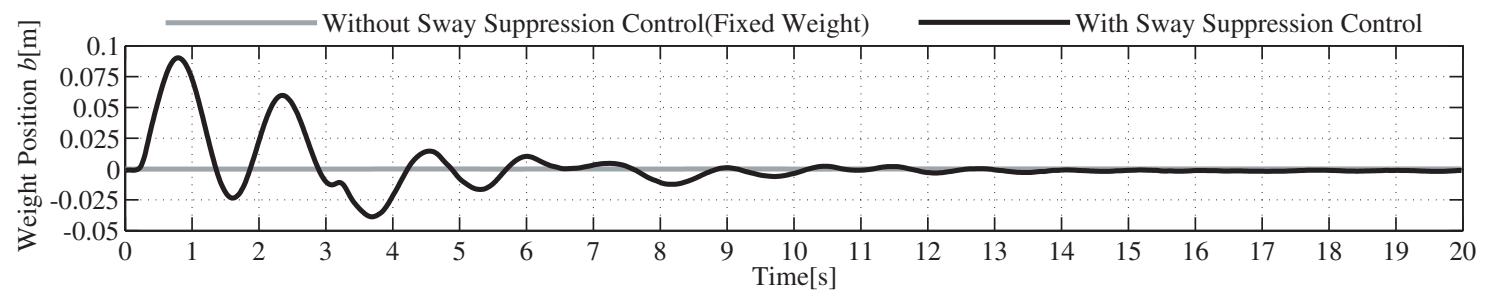

(b) Time Series Graph of Weight Position

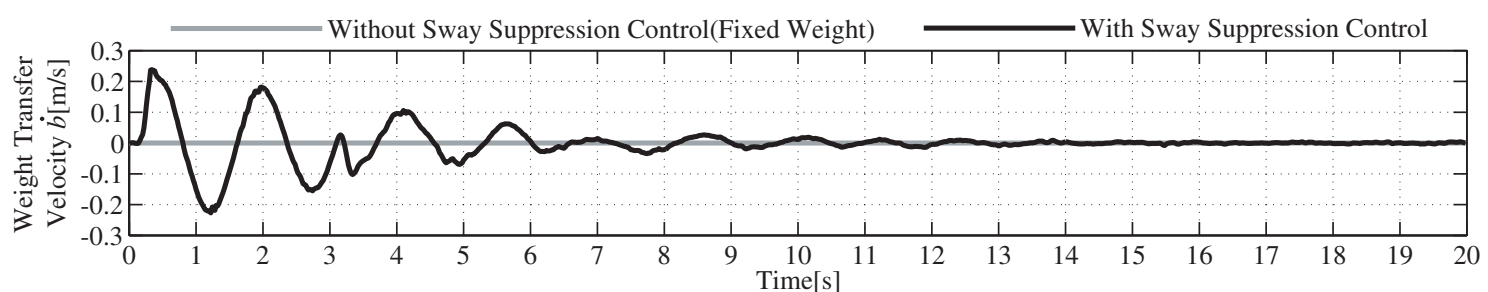

(c) Time Series Graph of Weight Transfer Velocity

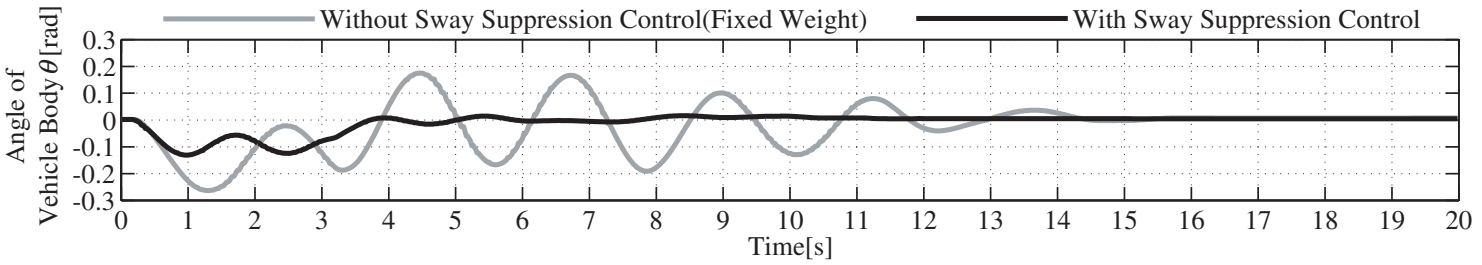

(d) Time Series Graph of Angle of Vehicle Body

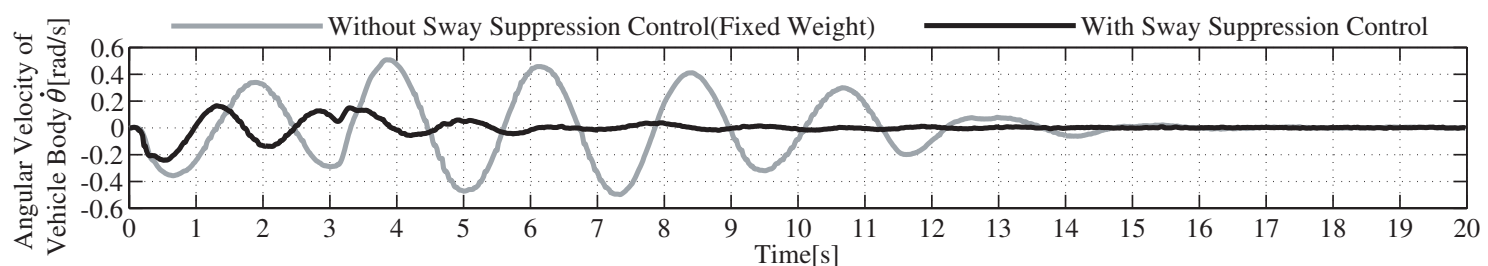

(e) Time Series Graph of Angular Velocity of Vehicle Body

Fig. 13 Experimental Results Comparison between Behaviors of Vehicle Body in Driven Active Mass System and Fixed Active Mass System

\section{References}

Ahmad, S., Siddique, N.H. and Tokhi, M.O., A modular fuzzy control approach for two-wheeled wheelchair, Journal of Intelligent and Robotic Systems: Theory and Applications, Vol. 64, Issue 3-4 (2011), pp.401-426.

Fu, J. and Zao, J., A new adaptive backstepping method for nonlinear control of turbine main steam valve, Journal of Control Theory and Applications, Vol. 5, No.1 (2007), pp.17-22.

Karkoub, M. and Parent, M., Modeling and non-linear feedback stabilization of a two-wheel vehicle, Journal of System and Control Engineering, Vol. 218, No.8 (2004), pp.675-686.

Kato, M., Okugawa, M., Kobayashi, Y. and Kojima, N., Attitude angle control by using air cylinder system for inverted pendulum type mobile robot with self-righting mechanism, Proceedings of the 54th Japan Joint Automatic Control Conference (2011), pp.63-68 (in Japanese).

Krsti, M. and Tsiotras, P., Inverse optimal stabilization of a rigid spacecraft, IEEE Transactions on Automatic Control, Vol. 44, Issue 5 (1999), pp.1042-1048.

Li, A. and Ando, R., Measuring the acceptability of self-balancing two-wheeled personal mobility vehicles, Journal of the 
Eastern Asia Society for Transportation Studies, Vol. 10 (2013), pp.444-453.

Lim, H., Yoshikawa, M. and Tamai, H., Development of a portable motor vehicle for personal transportation, Proceedings of International Conference on Control, Automation and Systems 2008 (2008), pp. 2742 - 2747.

Nakagawa, C., Nakano, K., Suda, Y. and Hirayama, Y., Stability of the two-wheeled inverted pendulum vehicle moved by human pedaling, Journal of System Design and Dynamics, Vol. 5, No. 3 (2011), pp.389-402.

Nelder, J. and Mead, R., A simplex method for function minimization. Computer Journal, Vol. 7 (1965), pp.308-313.

Noda, Y., Kawaguchi, A. and Terashima, K., A mechatronics vision for smart wheelchairs, Mobile Robots Navigation, IN-TECH Book (2010), pp.609-628.

Noda, Y., Sago, Y., Terashima, K., Kakihara, K. and Kawamura, H., Development of parallel two-wheel vehicle with lower gravity center of vehicle body, Proceedings of 9th International Conference on Informatics in Control, Automation and Robotics, Vol. 2 (2012), pp.70-76.

Rugh, W. and Shamma, J., Research on gain scheduling survey paper, Automatica, Vol. 36 (2000), pp.1401-1425.

Sasaki, M., Yanagihara, N., Matsumoto, O. and Komoriya, K., Steering control of the personal riding-type wheeled mobile platform (PMP), IEEE/RSJ International Conference on Intelligent Robots and Systems (2005) pp.3821-3826.

Shibata, K. and Ichii, H., Improvement of control performances of two-wheeled inverted pendulum using moving weight system, Proceedings of the 2013 JSME Conference on Robotics and Mechatronics (2013), pp.2A2-R11 (in Japanese).

Ueno, Y., Ohno, T., Terashima, K. and Kitagawa, H., The development of driving system with differential drive steering system for omni-directional mobile robot, Preprints of the IEEE International Conference on Mechatronics and Automation proceedings 2009 (2009), pp.1089-1094. 